\title{
Review: providing recordings or summaries of consultations may help patients with cancer
}

Scott JT, Entwistle VA, Sowden AJ, Watt I. Recordings or summaries of consultations for people with cancer. Cochrane

Review, latest version 14 Jul 1999. In: Cochrane Library. Oxford: Update Software.

\section{QUESTION: What is the effectiveness of providing recordings or summaries of consultations with practitioners to patients with cancer and their families?}

\section{Data sources}

Studies were identified by searching 20 databases, checking bibliographies of relevant studies, and contacting experts in the field.

\section{Study selection}

Studies were selected if they were randomised or non-randomised controlled trials that assessed the provision of recordings (audiotapes or videotapes) or written summaries of practitioner consultations to patients with cancer and their families.

\section{Data extraction}

Data were extracted on the consultations, type of medium used, delivery of recording or summary, and interventions used. The outcomes were information access, use, and understanding; experience of health care (satisfaction, participation in subsequent consultations, complaints, and litigation); and health and wellbeing.

\section{Main results}

8 randomised controlled trials ( $>664$ patients) were included. Heterogeneity among the studies prevented a meta-analysis from being done. 1 trial compared written summaries with usual care, 5 compared audiotapes with usual care, 1 compared audiotaped consultations with general cancer information tapes, and 1 compared audiotapes with summary letters. Of 6 studies that assessed information recall, 4 showed better recall among patients who received recordings or summaries than among those in control groups. Of 4 studies that assessed patient satisfaction with the consultation, 1 study showed that patients who received a written summary were more satisfied than those who did not receive a summary; another study showed that patients who received an audiotape of their consultation were more satisfied than those who received a general cancer information tape or no tape. 1 study showed that patients who received a tape of their consultation were more likely to ask questions in the subsequent consultation. No studies reported complaints or litigation. 6 studies that assessed anxiety or depression showed no differences between intervention and control groups. $96 \%$ of patients in 7 studies that provided data reported that tapes or written summaries were useful as reminders of what was discussed and to inform family members.

\section{Conclusions}

Providing recordings or summaries of consultations with practitioners helps patients with cancer to remem- ber what was discussed and thus better inform family members. Evidence for improvement in satisfaction and health and wellbeing is inconclusive.

\section{COMMENTARY}

The conclusions of this review by Scott $e t$ al are limited by the marked heterogeneity of the populations studied, unstated hypotheses, a paucity of intention to treat studies, and the possibility of inadequate sample sizes. The goal is laudable, however, because patients recall information poorly and benefit when information is provided effectively. ${ }^{1}$ Extensive guidelines for providing medical information, including bad news, have been published and include checking a patient's baseline understanding, encouraging questions, categorising information, and using patient centered communication and relational skills. ${ }^{2}$

The studies included in this review describe exciting, innovative approaches for enhancing recall, but we do not know whether other principles of effectively providing information were missed. For example, were the physicians systematically trained, and how patient centred were they, if at all? Was patient centred communication measured? A systematic patient centred method that includes patient education can be effectively learned and used by physicians. ${ }^{3}$

I am concerned that simply providing a tape or written summary to patients without attending to the other principles of patient education will not educate patients adequately. This concern is particularly relevant to patients with cancer because physicians are known to have more difficulty with patient education in such emotion laden situations. ${ }^{45}$

The published evidence suggests that the simple provision of tapes or summaries to patients assists in recall and informing family, but it is otherwise insufficient. It remains to be shown how such simple methods compare with alternative approaches to the complex, multidimensional task of patient education.

Robert C Smith, MD Michigan State University East Lansing, Michigan, USA

1 Ley P. Doctor-patient communication: some quantitative estimates of the role of cognitive factors in non-compliance. J Hypertens Suppl 1985;3:S51-5.

2 Smith RC. The patient's story:integrated patient-doctor interviewing. Boston: Little, Brown, 1996.

3 Langewitz WA, Eich P, Kiss A, et al. Improving communication skills-a randomized controlled behaviorally oriented intervention study for residents in internal medicine. Psychosom Med 1998;60:268-76.

4 Smith RC, Lyles JS, Mettler J, et al. The effectiveness of intensive training for residents in interviewing. Ann Intern Med 1998;128:118-26.

5 Maguire P, Faulkner A, Booth K, et al. Helping cancer patients disclose their concerns. Eur J Cancer 1996;32A:78-81. 